\title{
Impacto de una intervención de mejora de calidad de atención para prevenir las úlceras por presión en una Unidad de Cuidados Intensivos Pediátricos
}

\author{
Impact of a health care quality improvement intervention to \\ prevent pressure ulcers in a Pediatric Intensive Care Unit
}

\author{
Dra. Valeria Aprea ${ }^{a}$, Dr. Facundo Jorro Barón ${ }^{a}$, Dra. Claudia Meregalli ${ }^{a}$ y \\ Dra. María C. Sabatini
}

\section{RESUMEN}

Introducción. En los niños internados en las unidades de cuidadosintensivos, más del $50 \%$ de las úlceras por presión(UPP) se relacionan con la presión sostenida de los equipos y dispositivos. las UPP son un indicador indirecto de la calidad de atención.

Objetivo. Evaluar el impacto de la implementación de una intervención de mejora de la calidad de atención sobre el desarrollo de UPP en una Unidad de Cuidados Intensivos Pediátricos.

Material y métodos. Estudio antes-después, no controlado. Preintervención: medición de UPP; posintervención: se aplicó un paquete de medidas (educación del personal, identificación de los pacientes de riesgo y disminución de la presión mediante el uso de colchones antiescaras y posicionadores de gel de polímero), coniguales mediciones.

Resultados. Se incluyeron 152 pacientes, 74 preintervención y 78 posintervención. Se observó una disminución significativa de la incidencia de UPP (pre-: 50,60\%; pos-: 23,08\%; $p=0,001$ ). Mayor puntaje de riesgo en el grupo posintervención (pre-: 12,4 \pm 1 ,9; pos-: 13,7 $\pm 2,1$; $p=0,001)$. Sin diferencias en el puntaje de riesgo al día de aparición de la UPP y la cantidad de UPP. El análisis estratificado por riesgo mantuvo la diferencia significativa en la incidencia de UPP (PIM2 < 5: 47,37\%; 19,23\%; $p=0,004 ; \mathrm{y}$ PIM2 $\geq 5: 55,56 \% ; 30,77 \% ; p=0,053)$.

Servicio de Terapia Intensiva del Hospital General de Niños Dr. Pedro de Elizalde (HGNPE), Ciudad Autónoma de Buenos Aires, Argentina.

Correspondencia:

Dr. Facundo Jorro Barón: jorrobox@yahoo.com.ar

Financiamiento:

Ninguno.

Conflicto de intereses:

Ninguno que declarar.

Recibido: 11-9-2017

Aceptado: 8-2-2018

Cómo citar: Aprea V, Jorro Barón F, Meregalli C, Sabatini MC. Impacto de una intervención de mejora de calidad de atención para prevenir las úlceras por presión en una Unidad de Cuidados Intensivos Pediátricos. Arch Argent Pediatr 2018;116(4):e529-e541.

\section{INTRODUCCIÓN}

La piel es el tejido más extenso del cuerpo humano y provee una barrera protectora contra bacterias, sustancias químicas y físicas, mientras mantiene la homeostasis del medio interno. La piel recibe un tercio del volumen sanguíneo circulante y se encuentra involucrada en varias funciones, que incluyen protección, inmunidad, termorregulación, metabolismo, comunicación, identificación y sensaciones.

La lesión de la dermis o de la epidermis puede llevar a la producción de infecciones sistémicas, aumento de la morbilidad, mayores costos de atención e implicancias psicosociales negativas asociadas a las escaras y a la alopecia. Estas úlceras son áreas de necrosis focal en la piel y el tejido subyacente, causada por una interrupción del flujo sanguíneo en la zona afectada, como consecuencia de la presión prolongada entre una prominencia ósea y una superficie externa. ${ }^{1,2}$

El desarrollo de úlceras por presión (UPP) ha sido ampliamente estudiado en niños y adultos, y se lo ha descrito como un fenómeno que ocurre típicamente en pacientes adultos. Sin embargo, se han postulado factores de riesgo para el potencial desarrollo de UPP en pacientes pediátricos distintos a los observados en pacientes adultos. ${ }^{3}$

La población pediátrica y neonatal ingresada en unidades de cuidados críticos, unidades de hospitalización o aquellos niños con enfermedades 
crónicas, déficit psicomotor, problemas neurológicos o con lesiones medulares son los grupos de mayor riesgo y mayor incidencia de UPP. Se ha reportado una incidencia de UPP en pacientes pediátricos críticamente enfermos del $18 \%$ al $27 \%{ }^{4}$

En esta población de pacientes, el desarrollo de UPP se ha relacionado con distintas situaciones clínicas. ${ }^{5-8}$

Las UPP son un indicador indirecto de la calidad de atención y representan un creciente problema de salud debido a que afectan la calidad de vida del paciente, prolongan la estancia hospitalaria, aumentan la mortalidad y representan un alto costo económico para el sistema de salud.

\section{OBJETIVO}

El objetivo de este estudio fue evaluar el impacto de la implementación de una intervención de mejora de la calidad de atención sobre el desarrollo de UPP en una Unidad de Cuidados Intensivos Pediátricos (UCIP).

\section{MATERIALES Y MÉTODO Diseño}

Estudio antes-después, no controlado.

La investigación se desarrolló en dos etapas. En la primera etapa ("antes"), comprendida entre marzo y septiembre de 2014, se analizaron los datos basales. Sobre la base de los datos analizados, se plantearon las medidas de prevención. A partir del mes de abril de 2016, se aplicó una intervención de mejora de la calidad de atención para disminuir la incidencia de UPP en la Unidad (paquete de medidas aplicadas: véase el Anexo 1). En la segunda etapa ("después"), comprendida entre mayo y septiembre de 2016, se continuó con la intervención y se midieron sus resultados sobre la incidencia de UPP.

Al ingreso, se realizó, en todos los niños, la evaluación del riesgo de UPP por medio de la escala de Braden $Q$ y un examen exhaustivo, que incluyó la evaluación de la piel con examen a fondo de las zonas de alto riesgo, como debajo de férulas, aparatos ortopédicos, cánulas de traqueotomía, etc., y se documentaron los resultados en la ficha de recolección de datos.

Este procedimiento se repitió diariamente; si se observaban úlceras, se documentaba la ubicación, el tamaño y el estadio.

\section{Descripción del ámbito de estudio}

La investigación se realizó en la UCIP del Hospital General de Niños Pedro de Elizalde (HGNPE). Esta Unidad contaba con 11 camas médico-quirúrgicas, sin cirugía cardiovascular.

\section{Muestra}

Todos los niños mayores de un mes que ingresaron a la UCIP del HGNPE durante el período comprendido entre los meses de marzo y septiembre de 2014 y el período comprendido entre los meses de mayo y septiembre de 2016.

Criterios de exclusión:

- Estadía en la Unidad menor de 48 horas.

- Presencia de úlceras al momento del ingreso.

\section{Instrumento de recolección de datos}

La ficha de recolección de datos constaba de 3 partes (véase el Anexo 2):

- Datos epidemiológicos.

TABla 1: Composición de los distintos grupos de pacientes antes y después de la intervención

\begin{tabular}{lccc}
\hline Variable & Grupo preintervención & Grupo posintervención & $p$ \\
\hline Número de pacientes & 74 & 78 & \\
Porcentaje de ocupación & $80,6 \%$ & $91,3 \%$ & 0,169 \\
Peso (kg) & $8(5-15)$ & $7(4,7-10)$ & 0,119 \\
Edad (meses) & $8,5(3-36)$ & $6(3-20)$ & 0,738 \\
Días de ARM & $7(3-13)$ & $7(4-11)$ & 0,011 \\
PIM2 & $4,83(1,48-10,16)$ & $2,35(1,19-6,18)$ & 0,516 \\
Días de internación en la UTIP & $9(5-16)$ & $8(6-12)$ & \\
Diagnóstico: & & & \\
Respiratorio & $60 \%$ & $64,5 \%$ & \\
Cardiovascular & $0 \%$ & $3,8 \%$ & \\
Neurológico & $7 \%$ & $2,5 \%$ & \\
Sepsis & $14 \%$ & $13,9 \%$ & \\
Posquirúrgico & $10 \%$ & $7,6 \%$ & \\
Otros & $9 \%$ & $7,6 \%$ & \\
\hline
\end{tabular}

ARM: asistencia respiratoria mecánica; PIM2: Pediatric Index of Mortality 2; UTIP: Unidad de Terapia Intensiva Pediátrica. 
- Evaluación del riesgo de presentar UPP: se utilizó la escala de Braden $\mathrm{Q}^{9}$ al ingreso del niño a la Unidad de Cuidado Intensivo y en forma diaria (véase el Anexo 3).

- Clasificación de la UPP en estadios: mediante el sistema de 4 estadios del Grupo Nacional para el Estudio y Asesoramiento en Úlceras por Presión y Heridas Crónicas (GNEAUPP) ${ }^{5}$ (véase el Anexo 4).

\section{Análisis estadístico}

Los datos fueron presentados como media y desvío estándar o mediana e intervalo intercuartilo según distribución. Para comparar los datos antes y después, se utilizó el test $t$ de Student o Wilcoxon Rank Sum según la normalidad y el test de chi $^{2}$. Los datos se analizaron con el programa STATA 10.1 (Statistics/Data Analysis Stata Corporation 4905 Lakeway Drive College Station, TX, EE. UU.).

\section{Tamaño muestral}

Se calcularon 80 pacientes por etapa para identificar una diferencia mínima entre grupos de $20 \%$ (incidencia inicial de $40 \%$ vs. incidencia final menor del 20\%), con una potencia mayor del $80 \%$ y una $\mathrm{p}<0,05 \mathrm{y}$ pérdidas al seguimiento del $15 \%$.

\section{Consideraciones éticas}

En el presente estudio, no se realizó la identificación de los pacientes por su nombre o número de historia clínica; fueron codificados. El cuidado y prevención de UPP es un estándar de tratamiento en la UCIP a partir del mes de mayo de 2016. Debido a lo expuesto, no se consideró necesaria la solicitud de consentimiento informado.

\section{RESULTADOS}

Se incluyeron en el estudio 152 pacientes, 74 en el período preintervención y 78 en el período posintervención. La mediana de edad fue 7 (intervalo intercuartílico -IQ-: 4,7-27,5) meses. La mediana de Pediatric Index of Mortality 2 (PIM2) fue 3,71 (IQ: 1,29-8,18). La mediana de días de asistencia respiratoria mecánica (ARM) fue 7 (IQ: 4-7) días y la mediana de días de internación en la UCIP fue 8 (IQ: 6-14) días. No hubo diferencias significativas en la edad, peso, días de ARM, días de internación y diagnósticos de ingreso entre los grupos pre- y posintervención. Solo se observó un puntaje de gravedad, PIM2, mayor en el grupo preintervención [4,88 (1,36-10,7); 2,35 (1,19-6,18); $p=0,012]$ (Tabla 1).

La localización de UPP más frecuente fue en la región occipital baja, seguida de la maleolar externa y de la occipital alta (Figura 1).

Luego del período de intervención, se observó una disminución significativa de la incidencia de UPP (pre-: 50,60\%; pos-: 23,08\%; $p=0,001$ ). En el grupo preintervención, se observaron 81,9 UPP por 1000 días de internación, con 1 en estadio III y 7 en II, 70 en I; mientras que, en el grupo posintervención, se observaron

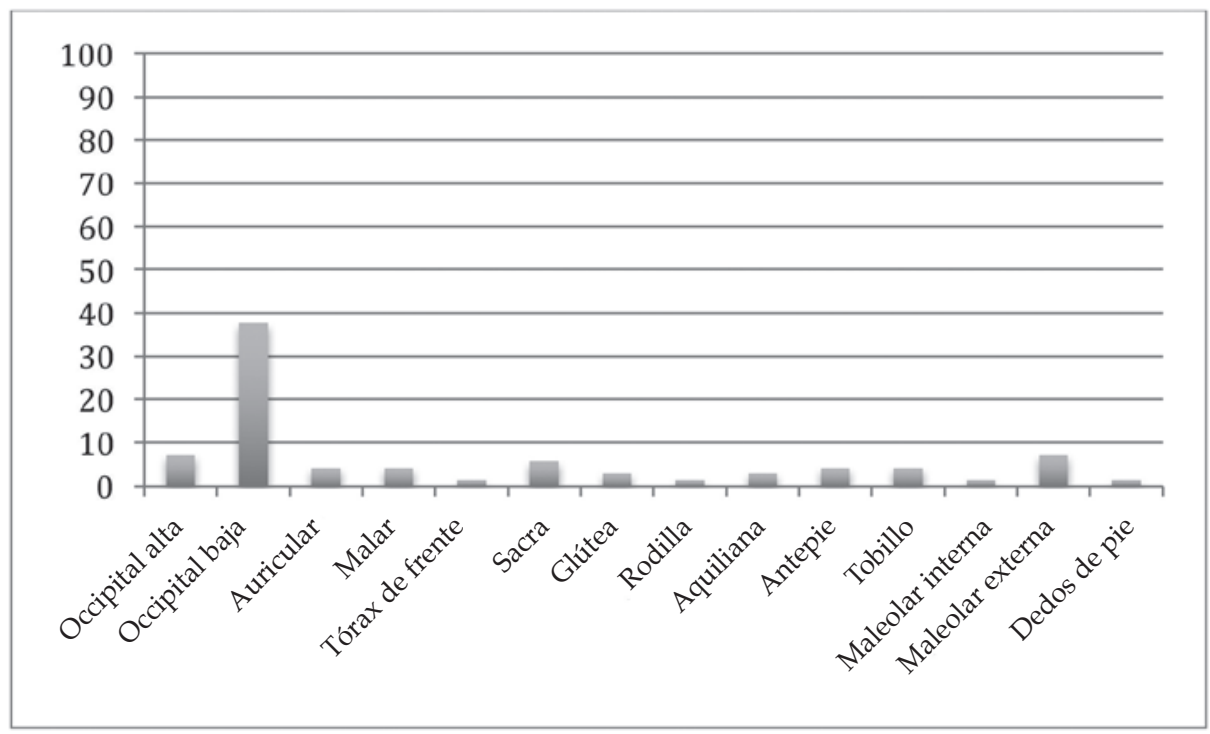


29,3 UPP por 1000 días de internación, ninguna en estadio III, 2 en II y 26 en I (Figura 2). El puntaje de riesgo para el desarrollo de UPP al inicio fue mayor en el grupo posintervención (pre-: $12,4 \pm 1,9$; pos-: $13,7 \pm 2,1 ; p=0,001$ ), y no se observaron modificaciones en el puntaje de riesgo al día de aparición de la UPP entre ambos grupos (pre- : $13,1 \pm 1,9$; pos-: $13,3 \pm 1,4 ; p=0,754$ ). La cantidad de UPP por paciente fue similar en ambos grupos (Tabla 2).

Por haberse registrado un PIM2 mayor en el grupo preintervención, se realizó un análisis estratificado por riesgo. Se dicotomizó el puntaje de PIM2 en $\geq 5$ y $<5$. Luego de la estratificación, se continuó observando una diferencia significativa en la incidencia de UPP (PIM2 < 5: 47,37\%; $19,23 \% ; p=0,004 ; \mathrm{y}$ PIM2 $\geq 5: 55,56 \% ; 30,77 \%$; $p=0,053)$.

\section{DISCUSIÓN}

Las UPP son eventos adversos frecuentes $\mathrm{y}$, muchas veces, prevenibles, asociados a los cuidados de la salud en pacientes hospitalizados. En la actualidad, son indicadores de calidad de atención sanitaria en pacientes críticos. La incidencia de UPP reportada en la población pediátrica varía entre el 10,2\% y el $33 \%{ }^{7,10-13} \mathrm{La}$ incidencia observada en la UCIP del HGNPE durante la primera etapa del estudio fue mayor que la informada por otros autores. Sin embargo, cuando se realiza la discriminación por estadio, algunos trabajos no incluyen el estadio I. En nuestra serie, se observó solo una UPP en estadio III en el período preintervención y ninguna en el posintervención. ${ }^{14}$ The National Quality Forum considera las UPP de estadio III, IV y no estadificables adquiridas luego de la admisión como un evento adverso serio prevenible. ${ }^{14}$

Dentro de los factores de riesgo asociados al desarrollo de UPP en niños críticamente enfermos, se encuentran la asistencia ventilatoria mecánica, la estadía en la UCIP mayor de 4 días, la necesidad de soporte inotrópico, el paro cardiorrespiratorio después de una cirugía cardiovascular, el uso de oxigenación por membrana extracorporal (extracorporeal membrane oxygenation; ECMO, por sus siglas en inglés), la inmovilidad, el déficit nutricional y la exposición

FIgURA 2: Cantidad y estadio de la úlcera por presión según el período

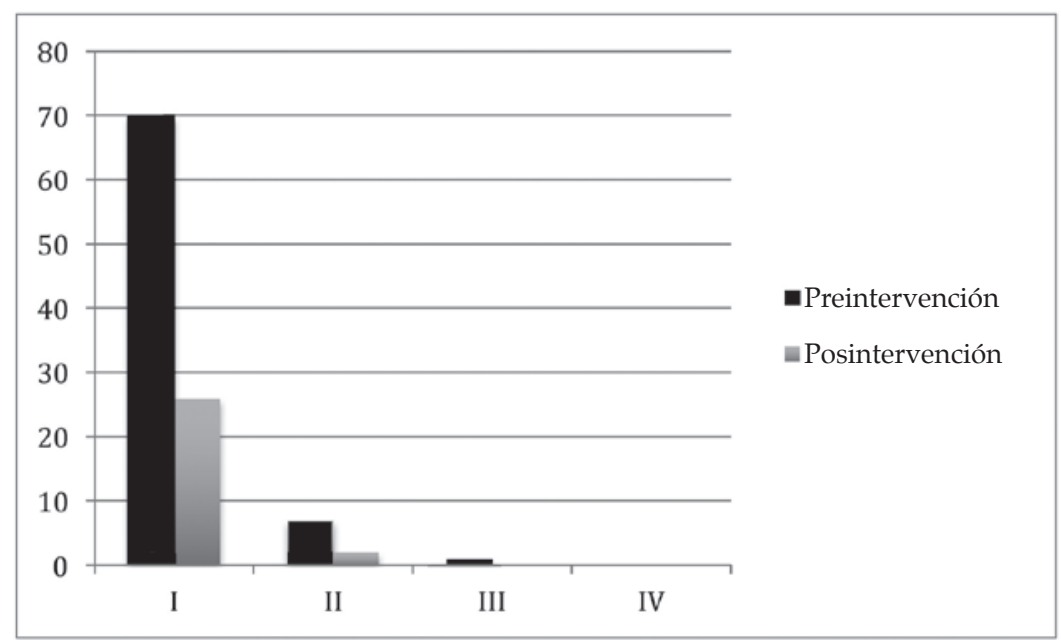

TABLA 2: Resultados de la intervención en los grupos de pacientes

\begin{tabular}{lccc}
\hline Variable & Grupo preintervención & Grupo posintervención & \\
\hline Escaras & $51,35 \%$ & $23,08 \%$ & $p=0,001$ \\
Puntaje de riesgo al inicio & $12(11-13)$ & $14(12-14)$ & $p=0,001$ \\
Puntaje de riesgo al día de inicio de la escara & $13(12-14)$ & $13(12-14)$ & $p=0,589$ \\
Día de aparición de la escara & $5(2-6,5)$ & $4(3-6)$ & $p=0,949$ \\
Estadio mayor de la escara & $1,1 \pm 0,37$ & $1,1 \pm 0,31$ & $p=0,477$ \\
Cantidad de escaras por paciente & $2,03 \pm 1,16$ & $1,55 \pm 0,69$ & $p=0,155$ \\
\hline
\end{tabular}


prolongada a dispositivos hospitalarios o invasividad con sondas y tubos (máscaras para ventilación no invasiva, cánulas de traqueotomía, tubos endotraqueales, entre otros). ${ }^{5,6,11,15}$ Una gran proporción de UPP (50-60\%) son asociadas a dispositivos hospitalarios en pediatría. ${ }^{3,16,17}$

La localización más frecuente de UPP en nuestro estudio fue en la región occipital baja, seguida por la región maleolar externa y la occipital superior. Este dato coincide con lo reportado en la literatura en niños menores de tres años. ${ }^{18}$

En nuestro Hospital, se aplicó un paquete de medidas para disminuir la incidencia de UPP, que incluyó educación del personal, identificación de los pacientes de riesgo y disminución de la presión mediante el uso de colchones antiescaras y posicionadores de gel de polímero. Luego de su aplicación, la incidencia de UPP disminuyó significativamente en el período posintervención, lo que coincidió con la bibliografía. ${ }^{3}$ No hubo diferencias significativas entre los grupos preintervención y posintervención en edad, peso, diagnóstico de ingreso, días de ARM ni días de internación. La única diferencia entre los grupos fue en el PIM2, que fue mayor en el grupo preintervención, motivo por el cual se realizó un análisis estratificado por riesgo. Se dicotomizó el puntaje de PIM2 en $\geq 5$ y $<5$. Luego de la estratificación, se continuó observando una diferencia significativa en la incidencia de UPP.

La implementación de programas de mejora continua de la calidad, que incluyan la capacitación del personal, la mejora de la comunicación, la identificación de los pacientes con mayor riesgo de padecer UPP y la estandarización de los procedimientos de atención y cuidado, ha demostrado disminuir la incidencia de UPP. 3,12,15,16,19

En pediatría, hay pocos estudios publicados en los cuales se implemente un paquete de medidas para reducir la incidencia de UPP. Entre ellos, se encuentra el de Visscher y col., ${ }^{3}$ quienes reportaron una disminución de la incidencia de UPP del 50\% (pre-: 14,3 x 1000 pacientes/día; pos-: 3,7 x 1000 pacientes / día, $p<0,05$ ) luego de la aplicación de un programa de mejora de la calidad que incluía la evaluación frecuente de la piel, educación y empoderamiento del personal. Los autores aplicaron este mismo programa en la Unidad de Cuidados Intensivos Neonatales, en la cual no lograron reducir la tasa de incidencia de UPP, fenómeno que explicaron como consecuencia de la incorporación de un nuevo modelo de sensor para saturometría en la Unidad, que coincidió con el período de medición.

Otro de los estudios es el de Schindler y col., ${ }^{15}$ quienes reportaron una disminución de la incidencia de UPP en una UCIP en EE. UU. del $18,8 \%$ al $6,8 \%$ luego de la aplicación de un programa de mejora de la calidad que incluía educación del personal, cambios de posición e inspecciones de la superficies de apoyo frecuentes y adecuación nutricional.

Boesch y col., ${ }^{16}$ diseñaron una estrategia para reducir la incidencia de UPP asociadas al uso de cánulas de traqueotomía. La estrategia consistía en evaluar frecuentemente la piel y el dispositivo, disminuir la humedad de su superficie y evitar la presión del dispositivo sobre la piel. Con la aplicación del paquete de medidas, disminuyeron la incidencia de UPP (pre-: 8,6\%; durante: $2,6 \%$; pos-: 0,3\%).

En adultos, los trabajos de mejora de la calidad para disminuir la incidencia de UPP también reportan resultados satisfactorios. . $^{12,18}$

Luego de la aplicación del programa de mejoras, no hubo cambios en el estadio mayor de las UPP en nuestra Unidad. Esto se diferencia de lo reportado por Tayyib y col., ${ }^{12}$ en dos unidades de cuidados intensivos (UCI) en Arabia, quienes evidenciaron una disminución del mayor estadio de las UPP en el período posintervención.

En nuestro estudio, no hubo diferencias significativas en los días de aparición de escaras en las dos etapas de la investigación ni en la cantidad de escaras por pacientes. Coyer y col., ${ }^{19}$ en una UCI de Australia, reportaron menor número de UPP por paciente y un desarrollo más tardío de estas durante la internación en la UCI.

Las limitaciones de esta investigación son, en primer lugar, que es un estudio de centro único. En segundo lugar, que no se puede descartar el cambio de comportamiento del personal que participó en la segunda parte de la investigación, debido a la conciencia de ser observado activamente (efecto Hawthorne). En tercer lugar, no existe certeza de que los resultados estén asociados a la intervención aplicada, dada la ausencia de un grupo de control. En cuarto lugar, los períodos de estudio no son exactamente coincidentes, a pesar de que ambos incorporaron la época invernal. En quinto lugar, si bien la intervención fue aplicada de forma completa, no se midió la adherencia al paquete de medidas. Finalmente, el estudio no evalúa la persistencia en el tiempo del beneficio hallado. 


\section{CONCLUSIÓN}

Se evidenció una reducción en el desarrollo de UPP en la UCIP del HGNPE, luego de la implementación de una intervención de mejora de la calidad de atención. No se observaron cambios en la cantidad ni en el estadio de mayor gravedad de las úlceras. Se observaron muy pocos episodios de UPP de estadio mayor de 2.

\section{REFERENCIAS}

1. Hagelgans NA. Pediatric skin care issues for the home care nurse. Pediatr Nurs 1993;19(5):499-507.

2. McLane KM, Bookout K, McCord S, et al. The 2003 national pediatric pressure ulcer and skin breakdown prevalence study: a multisite study. J Wound Ostomy Continence Nurs 2004;31(4):168-78.

3. Visscher M, King A, Nie AM, et al. A Quality-Improvement Collaborative Project to Reduce Pressure Ulcers in PICUs. Pediatrics 2013;131(6):e1950-60.

4. Curley MA, Quigley SM, Lin M. Pressure ulcers in pediatric intensive care: incidence and associated factors. Pediatr Crit Care Med 2003;4(3):284-90.

5. Grupo Nacional para el Estudioy Asesoramiento en Úlceras por Presión y Heridas Crónicas. Clasificación-Estadiaje de las Úlceras por Presión. Logroño: GNEAUPP; 2003.

6. Neidig JR, Kleiber C, Oppliger RA. Risk factors associated with pressure ulcers in the pediatric patient following open-heart surgery. Prog Cardiovasc Nurs 1989;4(3):99-106.

7. Schindler CA, Mikhailov TA, Fischer K, et al. Skin integrity in critically ill and injured children. Am J Crit Care 2007;16(6):568-74.

8. McCord S, McElvain V, Sachdeva R, et al. Risk factors associated with pressure ulcers in the pediatric intensive care unit. JWound Ostomy Continence Nurs 2004;31(4):179-83.
9. Pancorbo-Hidalgo PL, García-Fernández FP, SoldevillaÁgreda JJ, et al. Escalas e instrumentos de valoración del riesgo de desarrollar úlceras por presión. Logroño: GNEAUPP; 2009.

10. Groeneveld A, Anderson M, Allen S, et al. The prevalence of pressure ulcers in a tertiary care pediatric and adult hospital. J Wound Ostomy Continence Nurs 2004;31(3):108-20.

11. Schlüer AB, Schols JM, Halfens RJ. Risk and associated factors of pressure ulcers in hospitalized children over 1 yeard age. J Spec Pediatr Nurs 2014;19(1): 80-9.

12. Tayyib N, Coyer F, Lewis P. A two-arm cluster randomized control trial to determine the effectiveness of a pressure ulcer prevention Bundle for criticaly ill patients. J Nurs Scholarsh 2015;47(3):237-47.

13. Frank G, Walsh KE, Wooton S, et al. Impact of a Pressure Injury Prevention Bundle in the Solutions for Patient Safety Network. Pediatr Qual Saf 2017;(22):e013.

14. National Quality Forum. Serious Reportable Events in Healthcare - 2011 Update: A Consensus Report. Washington, DC:NQF; 2011.

15. Schindler CA, Mikhailov TA, Cashin SE, et al. Under pressure: preventing pressure ulcers in critically ill infants. J Spec Pediatr Nurs 2013;18(4):329-41.

16. Boesch RP, Myers C, Garrett T, et al. Prevention of tracheostomy-related pressure ulcers in children. Pediatrics 2012;129(3):e792-7.

17. Visscher MO, White CC, Jones JN, et al. Face masks for noninvasive ventilation: fit, excess skin hydration, and pressure ulcers. Respir Care 2015;60(11):1536-47.

18. Manning MJ, Gauvreau K, Curley MA. Factors associeted with occipital pressure ulcers in hospitalized infants and children. Am J Crit Care 2015;24(4):342-8.

19. Coyer F, Gardner A, Doubrovsky A, et al. Reducing pressure injuries in critically ill patients by using a patient skin integrity Care Bundle (INSPIRE). Am J Crit Care 2015;24(3):199-209. 


\section{ANEXO 1: PAQUETE DE MEDIDAS POR APLICAR}

1. PROGRAMA EDUCATIVO: se desarrollaron clases de 30 minutos de duración; participaron todos los médicos, enfermeros y kinesiólogos del Servicio.

Se incluyó información sobre los siguientes aspectos:

- La etiología y los factores de riesgo de las UPP.

- El uso de herramientas de evaluación, como la escala de Braden Q, para la predicción del riesgo de UPP.

- Utilización de categorías de evaluación del riesgo para determinar riesgos específicos y garantizar una planificación de cuidados eficaz.

- Evaluación de la piel.

- Clasificación de las UPP.

- Selección y / o uso de superficies de apoyo.

- Elaboración e implantación de un programa personalizado del cuidado de la piel.

- Demostración de cambios posturales y transferencia para reducir el riesgo de deterioro de los tejidos.

- Instrucciones sobre la documentación precisa de los datos pertinentes.

- Roles y responsabilidades de los miembros del equipo en relación con la evaluación y la prevención de las UPP.

- Cuidados de la piel.

- Cuidados de la piel:

- Se debe examinar el estado de la piel, al menos, una vez al día.

- Se debe mantener la piel del paciente en todo momento limpia y seca.

- Se deben utilizar jabones con pH neutro o sustancias limpiadoras con potencial irritativo bajo.

- Se debe lavar la piel con agua tibia, aclarar y realizar un secado meticuloso sin fricción.

- No se debe utilizar sobre la piel ningún tipo de alcohol (colonias, etc.).

- No se deben realizar masajes directamente sobre prominencias óseas.

- Se deben evaluar con detenimiento las zonas donde existieron lesiones por presión, dado que presentan un riesgo elevado de aparición de nuevas lesiones.

- Se deben controlar situaciones de humedad, pañales, drenajes y accesos vasculares.

- Se debe controlar la presión aplicando los siguientes principios: movilización, cambios posturales, utilización de superficies especiales de apoyo y protección local ante la presión.

- Se deben realizar cambios posturales cada 2 horas evitando cizallamientos y fricciones.

- Se debe redistribuir la presión en las zonas de riesgo: zona occipital, sacra, orejas, talones, nariz, etc.

- Se debe cambiar diariamente la ropa de cama y según sea necesario para mantenerla seca y sin arrugas.

- Se debe cambiar la localización de los dispositivos de monitoreo.

2. REDUCCIÓN DE LA PRESIÓN: mediante la adquisición de superficies especiales para el manejo de la presión; se realizó la compra de colchones antiescaras y posicionadores de gel de polímero.

Detalle de los nuevos materiales:

- 2 colchones de aire antiescaras con compresor regulable $190 \times 80$ × $6 \mathrm{~cm}$.

- 12 miniacomodadores para talón, $15 \times 8,3 \times 4,5 \mathrm{~cm}$.

- 2 almohadillas, redondas, huecas p/cabeza, pediátricas, $14 \mathrm{~cm}$ diámetro externo $\times 5,7 \mathrm{~cm}$ diámetro interno x 3,2 cm altura.

- 2 herraduras $\mathrm{p} /$ cabeza, pediátricas, $14 \mathrm{~cm}$ diámetro externo $\times 7,5 \mathrm{~cm}$ diámetro interno $\times 3,2 \mathrm{~cm}$ altura.

- 2 colchonetas para cadera/omóplato, 50,8 x 50,8 x 1,3 cm.

3. EVALUACIÓN DEL RIESGO DE DESARROLLAR UPP: se incorporó a la práctica diaria la utilización de la escala de Braden Q. Tuvo el siguiente objetivo:

- Identificar en forma precoz a los pacientes que pudieran presentar UPP.

- Proporcionar un criterio objetivo para la aplicación de medidas preventivas en función del nivel de riesgo.

- Asegurar la asignación eficiente y efectiva de recursos preventivos limitados. 


\section{ANEXO 2: HERRAMIENTA DE RECOLECCIÓN DE DATOS}

HOJA 1

NOMBRE:

EDAD:

PESO

DIAGNÓSTICO:

\section{ESCALA DE EVALUACIÓN DE RIESGO BRADEN Q}

\begin{tabular}{|c|c|c|c|c|}
\hline & $\begin{array}{l}\text { Intensidad y duración de la } \\
\text { presión } \\
\text { PUNTAJE }\end{array}$ & & & \\
\hline & MOVILIDAD & ACTIVIDAD & $\begin{array}{l}\text { PERCEPCIÓN } \\
\text { SENSORIAL }\end{array}$ & \\
\hline 1 & Completamente inmóvil & Encamado & $\begin{array}{l}\text { Completamente } \\
\text { limitada }\end{array}$ & \\
\hline 2 & Muy limitada & Sentado & Muy limitada & \\
\hline 3 & Ligeramente limitada & $\begin{array}{l}\text { Camina } \\
\text { ocasionalmente }\end{array}$ & Ligeramente limitada & \\
\hline 4 & Sin limitaciones & $\begin{array}{l}\text { Camina con } \\
\text { frecuencia }\end{array}$ & Sin limitaciones & \\
\hline & $\begin{array}{l}\text { Tolerancia de la piel y los } \\
\text { tejidos de soporte }\end{array}$ & & & \\
\hline & HUMEDAD & FRICCIÓN & NUTRICIÓN & PERFUSIÓN \\
\hline 1 & Constantemente húmedo & $\begin{array}{l}\text { Probablemente } \\
\text { importante }\end{array}$ & Muy pobre & $\begin{array}{l}\text { Extremadamente } \\
\text { comprometida }\end{array}$ \\
\hline 2 & Frecuentemente húmedo & Problemática & Inadecuada & Comprometida \\
\hline 3 & Ocasionalmente húmedo & $\begin{array}{l}\text { Problema } \\
\text { potencial }\end{array}$ & Adecuada & Adecuada \\
\hline 4 & Casi nunca & Sin problemas & Excelente & Excelente \\
\hline
\end{tabular}


PUNTUACIÓN: 16-23, en riesgo; 13-15, riesgo moderado; 10-12, riesgo alto; < 9, riesgo muy alto.

HOJA 2

Fecha de ingreso: ......................

Lesiones previas

\section{Observaciones}

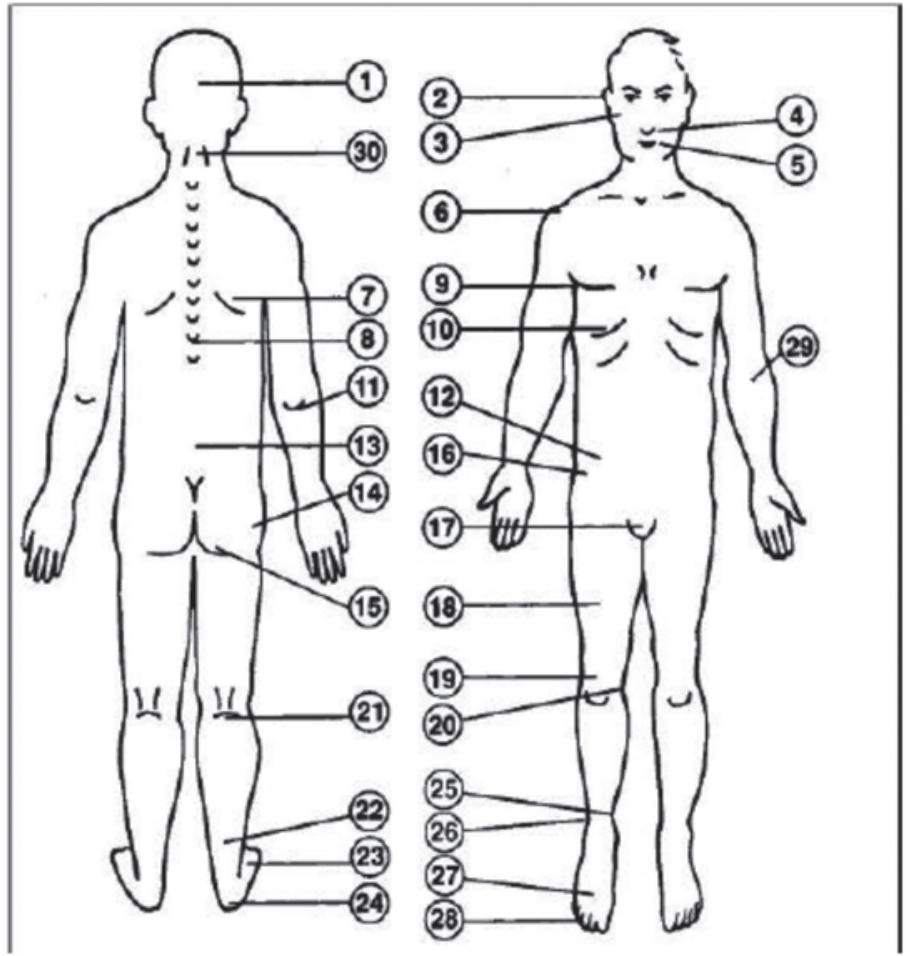

Ilustración 1. Localización de las úlceras por presión 


\section{ANEXO 3: ESCALA DE BRADEN Q}

\begin{tabular}{|c|c|c|c|c|c|}
\hline \multicolumn{5}{|c|}{ Intensidad y duración de la presión } & \multirow[t]{2}{*}{ Puntos } \\
\hline $\begin{array}{l}\text { MOVILIDAD } \\
\text { (capacidad para } \\
\text { cambiar y controlar } \\
\text { la posición del } \\
\text { cuerpo) }\end{array}$ & $\begin{array}{l}\text { 1. Completamente } \\
\text { inmóvil: No realiza ningún } \\
\text { movimiento con el cuerpo o } \\
\text { las extremidades sin ayuda. }\end{array}$ & $\begin{array}{l}\text { 2. Muy limitada: } \\
\text { Ocasionalmente, realiza } \\
\text { ligeros cambios en la } \\
\text { posición del cuerpo o de las } \\
\text { extremidades, pero no es } \\
\text { capaz de realizar cambios } \\
\text { frecuentes o significativos } \\
\text { sin ayuda. }\end{array}$ & $\begin{array}{l}\text { 3. Ligeramente limitada: } \\
\text { Realiza frecuentes aunque } \\
\text { ligeros cambios en la } \\
\text { posición del cuerpo o de las } \\
\text { extremidades sin ayuda. }\end{array}$ & $\begin{array}{l}\text { 4. Sin limitaciones: Realiza } \\
\text { importantes y frecuentes } \\
\text { cambios de posición sin } \\
\text { ayuda. }\end{array}$ & \\
\hline $\begin{array}{l}\text { ACTIVIDAD (nivel de } \\
\text { actividad fisica) }\end{array}$ & $\begin{array}{l}\text { 1. Encamado: Limitado/a a } \\
\text { la cama. }\end{array}$ & $\begin{array}{l}\text { 2. En silla: Capacidad para } \\
\text { caminar gravemente } \\
\text { limitada o inexistente. No } \\
\text { puede soportar su propio } \\
\text { peso y/o hay que ayudarlo } \\
\text { para sentarse en una silla o } \\
\text { en una silla de ruedas. }\end{array}$ & $\begin{array}{l}\text { 3. Camina } \\
\text { ocasionalmente: Camina } \\
\text { ocasionalmente durante el } \\
\text { dia, pero distancias muy } \\
\text { cortas, con o sin ayuda. } \\
\text { Pasa la mayor parte de } \\
\text { cada turno en la cama o en } \\
\text { la silla. }\end{array}$ & $\begin{array}{l}\text { 4. Todos los pacientes } \\
\text { demasiado jóvenes para } \\
\text { caminar o camina } \\
\text { frecuentemente. } \\
\text { Camina fuera de la } \\
\text { habitación, al menos, dos } \\
\text { veces al dia y dentro de la } \\
\text { habitación, al menos, una } \\
\text { vez cada dos horas durante } \\
\text { las horas de paseo. }\end{array}$ & \\
\hline $\begin{array}{l}\text { PERCEPCIÓN } \\
\text { SENSORIAL } \\
\text { (capacidad de } \\
\text { responder } \\
\text { adecuadamente, } \\
\text { según su nivel de } \\
\text { desarrollo, a la } \\
\text { incomodidad } \\
\text { relacionada con la } \\
\text { presión) }\end{array}$ & $\begin{array}{l}\text { 1. Completamente } \\
\text { limitada: No responde a } \\
\text { estimulos dolorosos (no } \\
\text { gime, no se estremece ni se } \\
\text { agarra), debido a un bajo } \\
\text { nivel de conciencia o a la } \\
\text { sedación. } \\
\text { O capacidad limitada para } \\
\text { sentir dolor en la mayor } \\
\text { parte del cuerpo. }\end{array}$ & $\begin{array}{l}\text { 2. Muy limitada: Responde } \\
\text { solo a estimulos dolorosos. } \\
\text { Incapacidad para comunicar } \\
\text { malestar, excepto gimiendo } \\
\text { o mostrando inquietud, o } \\
\text { tiene alguna alteración } \\
\text { sensorial que limita la } \\
\text { capacidad de sentir dolor o } \\
\text { malestar en más de la mitad } \\
\text { del cuerpo. }\end{array}$ & $\begin{array}{l}\text { 3. Ligeramente limitada: } \\
\text { Responde a órdenes } \\
\text { verbales, pero no siempre } \\
\text { puede comunicar la } \\
\text { incomodidad o la necesidad } \\
\text { de ser cambiado de postura. } \\
\text { O tiene alguna alteración } \\
\text { sensorial que limita la } \\
\text { capacidad de sentir dolor o } \\
\text { malestar en una o dos } \\
\text { extremidades. }\end{array}$ & $\begin{array}{l}\text { 4. Sin limitaciones: } \\
\text { Responde a órdenes } \\
\text { verbales. No tiene ninguna } \\
\text { alteración sensorial que } \\
\text { limite su capacidad de sentir } \\
\text { o comunicar dolor o } \\
\text { malestar. }\end{array}$ & \\
\hline \multicolumn{6}{|c|}{ Tolerancia de la piel y la estructura de soporte } \\
\hline $\begin{array}{l}\text { HUMEDAD (nivel de } \\
\text { exposición de la piel } \\
\text { a la humedad) }\end{array}$ & $\begin{array}{l}\text { 1. Piel constantemente } \\
\text { húmeda: La piel se } \\
\text { mantiene húmeda casi } \\
\text { permanentemente debido a } \\
\text { la transpiración, orina, } \\
\text { drenaje, etc. La humedad } \\
\text { es detectada cada vez que } \\
\text { se gira o mueve al paciente. }\end{array}$ & $\begin{array}{l}\text { 2. Piel muy húmeda: La } \\
\text { piel está húmeda a menudo, } \\
\text { pero no siempre. La ropa de } \\
\text { cama debe ser cambiada, al } \\
\text { menos, cada } 8 \text { horas. }\end{array}$ & $\begin{array}{l}\text { 3. Piel ocasionalmente } \\
\text { húmeda: La piel está } \\
\text { húmeda de forma ocasional, } \\
\text { por lo que necesita el } \\
\text { cambio de la ropa de cama } \\
\text { cada } 12 \text { horas. }\end{array}$ & $\begin{array}{l}\text { 4. Piel raramente húmeda: } \\
\text { La piel está casi siempre } \\
\text { seca. Hay que cambiar los } \\
\text { pañales de forma rutinaria; } \\
\text { la ropa de cama solo hay } \\
\text { que cambiarla cada } 24 \\
\text { horas. }\end{array}$ & \\
\hline
\end{tabular}




\begin{tabular}{|c|c|c|c|c|}
\hline $\begin{array}{l}\text { FRICCIÓN Y } \\
\text { CIZALLAMIENTO } \\
\text { (fricción: ocurre } \\
\text { cuando la piel se } \\
\text { mueve contra las } \\
\text { superficies de apoyo. } \\
\text { Cizallamiento: ocurre } \\
\text { cuando la piel y la } \\
\text { superficie ósea } \\
\text { adyacente rozan una } \\
\text { contra la otra) }\end{array}$ & $\begin{array}{l}\text { 1. Problema } \\
\text { significativo: La } \\
\text { espasticidad, } \\
\text { contracturas, picores o } \\
\text { agitación llevan a un } \\
\text { movimiento y fricción } \\
\text { casi constantes. }\end{array}$ & $\begin{array}{l}\text { 2. Problema: Requiere ayuda } \\
\text { de moderada a máxima para } \\
\text { moverse. Es imposible } \\
\text { levantarlo completamente sin } \\
\text { que se produzca un roce } \\
\text { contra las sábanas. Con } \\
\text { frecuencia, se desliza hacia } \\
\text { abajo en la cama o en la silla, } \\
\text { por lo que se necesita } \\
\text { recolocarlo con la máxima } \\
\text { ayuda. }\end{array}$ & $\begin{array}{l}\text { 3. Problema potencial: Se } \\
\text { mueve débilmente o necesita } \\
\text { una ayuda mínima. Mientras se } \\
\text { mueve, es posible que la piel } \\
\text { roce de algún modo con las } \\
\text { sábanas, la silla u otros } \\
\text { dispositivos. La mayor parte del } \\
\text { tiempo, mantiene una posición } \\
\text { relativamente buena en la silla o } \\
\text { cama, aunque, en ocasiones, se } \\
\text { desliza hacia abajo. }\end{array}$ & $\begin{array}{l}\text { 4. Sin problema aparente: } \\
\text { Es posible levantarlo } \\
\text { completamente durante un } \\
\text { cambio de postura. Se } \\
\text { mueve de modo } \\
\text { independiente en la cama y } \\
\text { en la silla, y tiene suficiente } \\
\text { fuerza muscular para } \\
\text { levantarse completamente } \\
\text { mientras se mueve. }\end{array}$ \\
\hline $\begin{array}{l}\text { NUTRICIÓN (patrón } \\
\text { habitual de ingesta } \\
\text { de alimentos) }\end{array}$ & $\begin{array}{l}\text { 1. Muy pobre: Está en } \\
\text { ayunas o a dieta } \\
\text { liquida o con } \\
\text { sueroterapia más de } 5 \\
\text { días. O albúmina < 2,5 } \\
\text { mg/dl o nunca come } \\
\text { una comida completa. } \\
\text { Raras veces, come } \\
\text { más de la mitad de } \\
\text { cualquier comida } \\
\text { ofrecida. La ingesta de } \\
\text { proteínas incluye } 20 \\
\text { menos raciones de } \\
\text { carne, pescado o } \\
\text { productos lácteos al } \\
\text { día. Toma pocos } \\
\text { líquidos. No toma un } \\
\text { suplemento dietético } \\
\text { liquido. }\end{array}$ & $\begin{array}{l}\text { 2. Inadecuada: Se le } \\
\text { administra una nutrición } \\
\text { enteral (sonda nasogástrica } \\
\text { _SNG_-) o nutrición } \\
\text { parenteral (intravenosa) que } \\
\text { le proporciona una cantidad } \\
\text { inadecuada de calorías y } \\
\text { minerales para su edad. O } \\
\text { albúmina < } 3 \text { mg/dl. O, raras } \\
\text { veces, come una comida } \\
\text { completa y, generalmente, } \\
\text { solo come la mitad de } \\
\text { cualquier comida ofrecida. La } \\
\text { ingesta de proteínas incluye } \\
\text { solo } 3 \text { raciones de carne, } \\
\text { pescado o productos lácteos } \\
\text { al dia. Ocasionalmente, toma } \\
\text { un suplemento dietético. }\end{array}$ & $\begin{array}{l}\text { 3. Adecuada: Se le administra } \\
\text { una nutrición enteral (SNG) o } \\
\text { nutrición parenteral } \\
\text { (intravenosa) que le proporciona } \\
\text { una cantidad adecuada de } \\
\text { calorías y minerales para su } \\
\text { edad. O come la mitad de la } \\
\text { mayoría de las comidas. Come } \\
\text { un total de } 4 \text { raciones de } \\
\text { proteinas al día (carne, } \\
\text { pescado, productos lácteos). En } \\
\text { ocasiones, rechaza una comida, } \\
\text { pero normalmente toma un } \\
\text { suplemento si se le ofrece. }\end{array}$ & $\begin{array}{l}\text { 4. Excelente: Toma una } \\
\text { dieta normal que le } \\
\text { proporciona las calorias } \\
\text { adecuadas para su edad. } \\
\text { Por ejemplo: come/bebe la } \\
\text { mayor parte de cada } \\
\text { comida/toma. Nunca } \\
\text { rechaza una comida. } \\
\text { Normalmente, come un } \\
\text { total de } 4 \text { o más raciones } \\
\text { de carne, pescados o } \\
\text { productos lácteos al dia. En } \\
\text { ocasiones, come entre } \\
\text { comidas. No necesita } \\
\text { suplementos. }\end{array}$ \\
\hline $\begin{array}{l}\text { PERFUSIÓN } \\
\text { TISULAR Y } \\
\text { OXIGENACIÓN }\end{array}$ & $\begin{array}{l}\text { 1. Muy } \\
\text { comprometida: } \\
\text { Hipotenso (tensión } \\
\text { arterial media }-\mathrm{TA}_{\mathrm{m}}- \\
<50 \mathrm{mmHg} ;<40 \mathrm{en} \\
\text { recién nacidos). O el } \\
\text { paciente no tolera } \\
\text { fisiológicamente los } \\
\text { cambios posturales. }\end{array}$ & $\begin{array}{l}\text { 2. Comprometida: } \\
\text { Normotenso; el pH sérico es } \\
<7,40 \text {; la saturación de } \\
\text { oxígeno puede ser }<95 \% \text { o la } \\
\text { hemoglobina puede ser }<10 \\
\text { mg/dl o el relleno capilar } \\
\text { puede ser }>2 \text { segundos. }\end{array}$ & $\begin{array}{l}\text { 3. Adecuada: Normotenso; el } \\
\text { pH de la sangre es normal; la } \\
\text { saturación de oxigeno puede } \\
\text { ser < } 95 \% \text {. O la hemoglobina } \\
\text { puede ser < } 10 \mathrm{mg} / \mathrm{dl} \text { o el } \\
\text { relleno capilar puede ser > } 2 \\
\text { segundos. }\end{array}$ & $\begin{array}{l}\text { 4. Excelente: Normotenso; } \\
\text { la saturación de oxígeno es } \\
>95 \% \text {; hemoglobina } \\
\text { normal y el relleno capilar < } \\
2 \text { segundos. }\end{array}$ \\
\hline
\end{tabular}

PUNTUACIÓN: 16-23, en riesgo; 13-15, riesgo moderado; 10-12, riesgo alto; < 9, riesgo muy alto.

(La escala Braden $Q$ fue desarrollada para la identificación del riesgo de úlceras por presión en niños de 21 días a 8

años. Contiene las 6 subescalas originales de la escala de Braden para adultos y una séptima subescala para la oxigenación tisular y perfusión. El punto de corte es de 16 puntos para determinar a los pacientes en riesgo). 


\section{ANEXO 4: CLASIFICACIÓN DE LAS ÚLCERAS POR PRESIÓN*}

Grupo Nacional para el Estudio y Asesoramiento en Úlceras por Presión y Heridas Crónicas (GNEAUPP). Clasificación en Estadios de las Úlceras por Presión.

\section{ESTADIO I}

Alteración observable en la piel íntegra, relacionada con la presión, que se manifiesta por un eritema cutáneo que no palidece al presionar; en pieles oscuras, puede presentar tonos rojos, azules o morados.

En comparación con un área (adyacente u opuesta) del cuerpo no sometida a presión, puede incluir cambios en uno o más de los siguientes aspectos:

- Temperatura de la piel (caliente o fría)

- Consistencia del tejido (edema, induración)

- $\quad$ Ylo sensaciones (dolor, escozor)

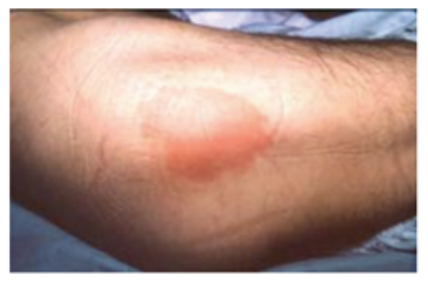

\section{ESTADIO II}

- Pérdida parcial del grosor de la piel que afecta a la epidermis, dermis o ambas.

- Úlcera superficial que tiene aspecto de abrasión, ampolla o cráter superficial.

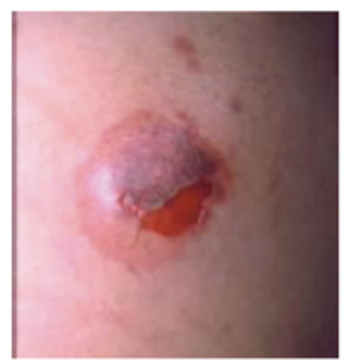




\section{ESTADIO III}

- Pérdida total del grosor de la piel que implica lesión o necrosis del tejido subcutáneo, que puede extenderse hacia abajo, pero no por la fascia subyacente.

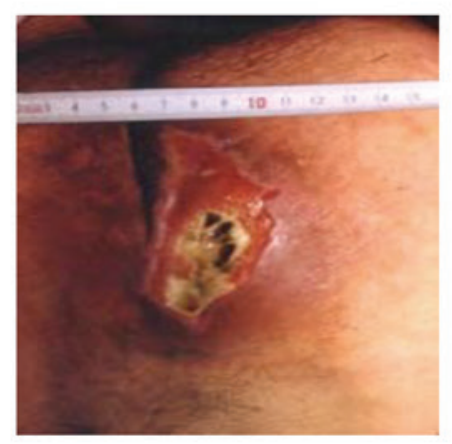

\section{ESTADIO IV}

- Pérdida total del grosor de la piel con destrucción extensa, necrosis del tejido o lesión en el músculo, el hueso o las estructuras de sostén (tendón, cápsula articular, etc.).

- En este estadio, como en el III, pueden presentarse lesiones con cavernas, tunelizaciones o trayectos sinuosos.

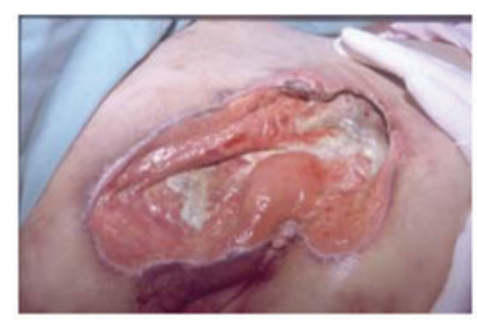

En todos los casos que procedan, deberá retirarse el tejido necrótico antes de determinar el estadio de la úlcera.

\footnotetext{
*Fotos extraídas, con autorización para su uso docente, del Grupo Nacional para el Estudio y Asesoramiento en Úlceras por Presión y Heridas Crónicas (GNEAUPP). Clasificación-Estadiaje de las Úlceras por Presión. Logroño. 2003. http://gneaupp.info/seccion/banco-de-imagenes/.
} 